\title{
Dynamic mitral regurgitation treated with MitraClip
}

\section{Dear Editor,}

The dynamic nature of mitral regurgitation (MR) has been well appreciated but clinically under-recognised. In addition, evidence on therapeutic options for dynamic MR has been lacking. We report the case of a 48-yearold woman who underwent coronary revascularisation and extra-corporeal membrane oxygenation (ECMO) support after post-operative cardiac collapse from left main (LM) coronary artery occlusion. However, she had difficulty coming off the ventilator due to recurrent pulmonary oedema from dynamic MR. She was treated with percutaneous mitral valve repair using the MitraClip system (Abbott Vascular, Santa Clara, US) and was successfully discharged.

The 48-year-old woman with right breast cancer was admitted for mastectomy and breast reconstruction. She had no past medical history. Post-operatively, she developed acute chest pain and hypotension. Electrocardiogram showed anterior ST-segment elevation, and the cardiac catheterisation laboratory was activated.

During the procedure, she had recurrent collapse from ventricular arrhythmias requiring cardiopulmonary resuscitation and repeated defibrillation. Intra-aortic balloon pump (IABP) was inserted and coronary angiography showed isolated LM coronary artery occlusion (Figs. 1A and 1B). After aspiration thrombectomy, a $3.5 \times 18 \mathrm{~mm}$ stent was implanted from LM into proximal left anterior descending (LAD) artery (Figs. 1C and 1D) establishing Thrombolysis in Myocardial Infarction (TIMI) 3 flow.

In view of haemodynamic and electrical instability, ECMO was implanted. Transthoracic echocardiogram (TTE) showed severely depressed left ventricular ejection fraction (LVEF) of 15-20\% with trivial MR. She was transferred back to the intensive care unit (ICU) and her stay was complicated by acute kidney injury requiring renal replacement therapy, abdominal haematoma requiring surgical evacuation and pneumonia. Inotropes were weaned off gradually and the ECMO was successfully explanted on day 8 of myocardial infarction. In view of the prolonged stay and ventilation required, she underwent a tracheostomy. However, despite adequate ultrafiltration, she had recurrent acute pulmonary oedema and was unable to come off the ventilator. She developed hypotension again requiring inotropes to be reinstated. Repeat TTE showed an improved LVEF of 25-30\% with moderate MR.

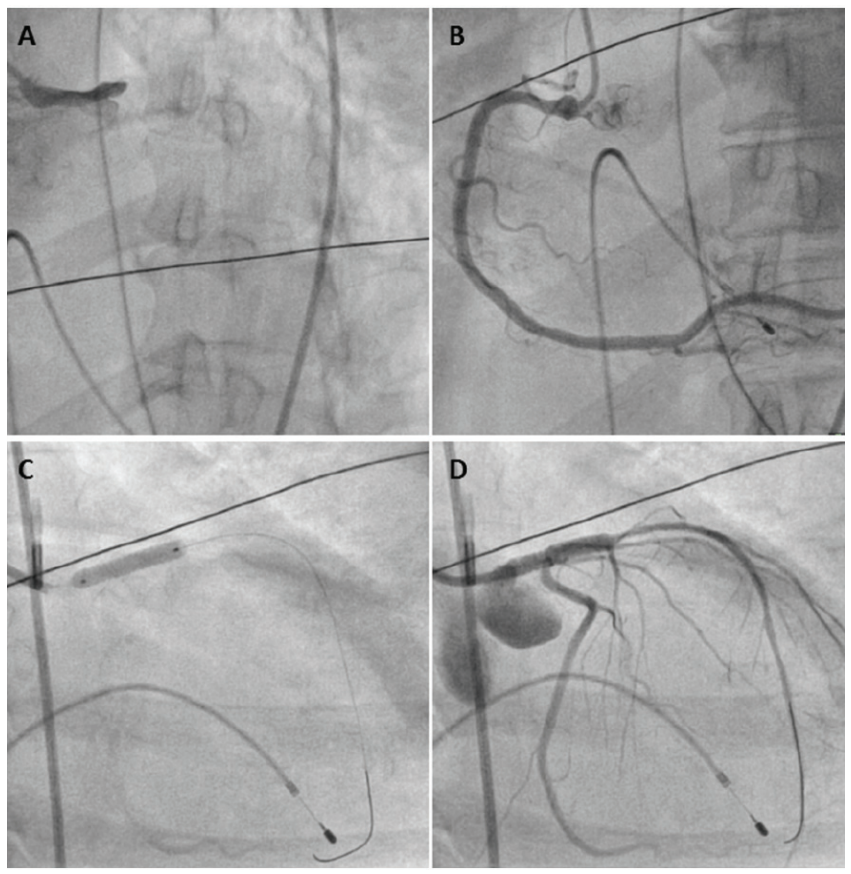

Fig 1. Fluoroscopic images showing (A) left main (LM) coronary artery occlusion with (B) normal right coronary artery. (C) $3.5 \times 18 \mathrm{~mm}$ stent implanted from LM into proximal left anterior descending (LAD) artery. (D) Final angiography showing patent LM, LAD and left circumflex arteries.

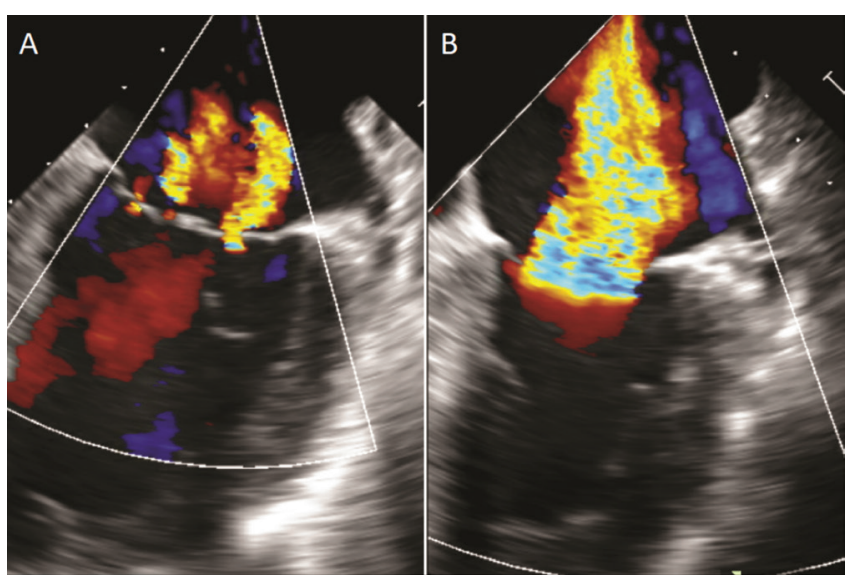

Fig. 2. Transesophageal echocardiogram images showing (A) moderate mitral regurgitation (MR) at systolic blood pressure (SBP) of $82 \mathrm{mmHg}$, which worsened to (B) severe MR at SBP of $130 \mathrm{mmHg}$. 
Duplex of the renal arteries did not reveal any stenosis. Transesophageal echocardiogram (TEE) was done in the ICU to better evaluate the mitral valve. The MR aetiology was functional with normal mitral valve morphology but tenting of the leaflets and a dilated annulus of $3.5 \mathrm{~cm}$. At systolic blood pressure (SBP) of $82 \mathrm{mmHg}$, there was moderate central $\mathrm{MR}$ and a pulmonary artery systolic pressure (PASP) of $47 \mathrm{mmHg}$ (Fig. 2A). However, the patient became agitated transiently and SBP went up to $130 \mathrm{mmHg}$ with increase in MR to severe and PASP to $79 \mathrm{mmHg}$ (Fig. 2B). Repeat coronary angiography was done, which showed patent LM to LAD stent, and IABP was re-inserted.

A Heart Team discussion including critical care physicians proposed that the severe functional MR was causing recurrent pulmonary oedema and haemodynamic compromise. Her European System for Cardiac Operative Risk Evaluation (EUROSCORE) II and Society of Thoracic Surgeons (STS) scores were $42.5 \%$ and $50.0 \%$ respectively. She was deemed to be at high risk for open-heart surgery, and hence decision was made for percutaneous edge-to-edge repair of the mitral valve with the MitraClip system. MitraClip was performed with implantation of 2 clips. First clip was placed slightly medial in $\mathrm{A} 2 / \mathrm{P} 2$ region with reduction of MR to 2+ (Figs. 3A and 3B). Second clip was placed just lateral to the first clip with reduction of MR to $1+$ (Fig. 3C), systolic predominance on pulmonary venous flow (Fig. 3D) and a trans-mitral mean pressure gradient of $3 \mathrm{mmHg}$ at SBP $110 \mathrm{mmHg}$. Following the procedure, her IABP was removed and she was successfully weaned off inotropes and ventilator. She was debilitated from the events, and stayed another 2 months in the hospital for rehabilitation. She made good progress with decannulation of the tracheostomy tube and achieved renal recovery without the need for long-term dialysis. She was discharged home and was able to walk with a walking frame.

MR severity at 1 year remained mild-to-moderate (Fig. 4). Unfortunately, she declined implantable cardioverter defibrillation implantation. She collapsed from ventricular tachycardia, which led to another long 4-month admission complicated by heart failure, sepsis from pneumonia and fungaemia, and prolonged intubation requiring another tracheostomy. Eventually, she and her family opted for a palliative route, and she died peacefully at home, one and a half years after MitraClip implantation.

Dynamic MR has been shown to be associated with poor outcomes and exercise capacity. ${ }^{1-3}$ In diseased ventricles, the delicate balance of tethering and closing

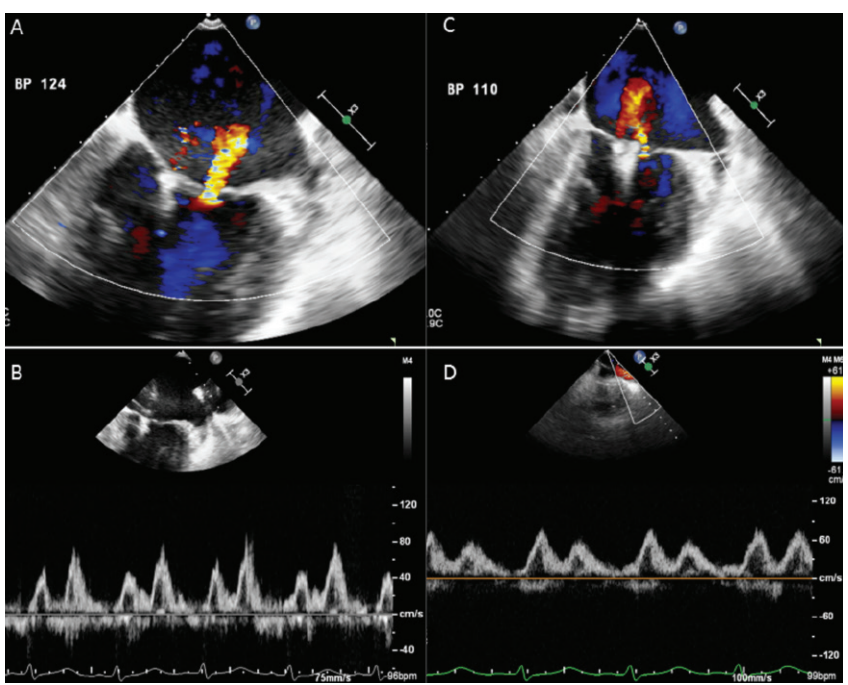

Fig. 3. Transesophageal echocardiogram images showing (A) first clip deployed slightly medial in A2/P2 region with residual lateral 2+ mitral regurgitation (MR) and (B) systolic blunting in pulmonary venous flow. (C) Second clip deployed just lateral to the first clip with reduction of MR to $1+$ at systolic blood pressure $110 \mathrm{mmHg}$ and (D) systolic predominance in pulmonary venous flow.
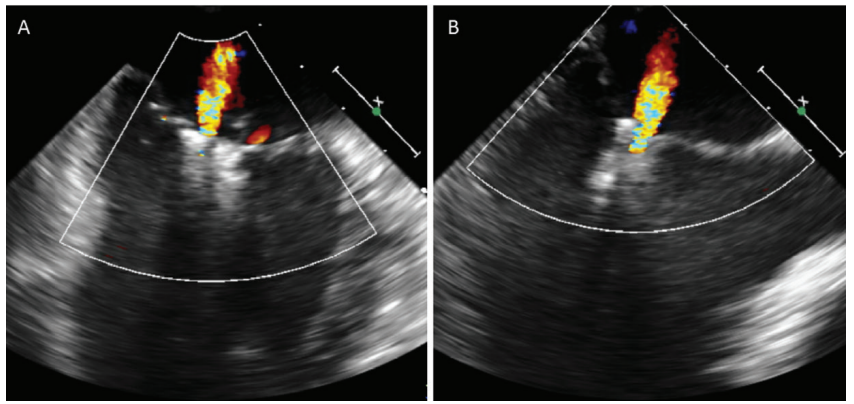

Fig. 4. Transesophageal echocardiogram images showing mild-tomoderate mitral regurgitation 1 year after MitraClip implantation in the (A) intercommissural and (B) left ventricular outflow tract views.

forces that is necessary for optimal mitral valve leaflet coaptation is often disrupted, resulting in MR. Both volume overload and increase in afterload, provoked either physiologically (exercise) or pharmacologically (vasopressors or fluid infusion), can result in further alterations in left ventricular geometry and hence, worsening of MR. This translates to a rapid rise in left atrial and pulmonary pressures, leading to the development of pulmonary oedema. However, it can be a challenge to recognise dynamic MR as the cause of symptoms and a high index of clinical suspicion is required. In our case, after excluding other causes of recurrent acute pulmonary oedema (i.e. ischaemia, renal artery stenosis), it was important to observe for dynamic change in MR severity in concordance with changes in blood pressure. 
There is a lack of data on effective therapies for dynamic MR. Extrapolation from treatment options for secondary MR has been made based on their individual targets on geometric determinants for dynamic MR. ${ }^{4}$ However, how each therapy, or a combination therapies directly impact symptoms and outcomes in this group of patients remains unclear. There are only a few reports of percutaneous repair with the MitraClip system for dynamic MR., 5 Our case demonstrated its successful use in a critically ill patient with recurrent acute pulmonary oedema from dynamic MR who was at prohibitive risk for surgery and its sustained impact at 1 year.

Lastly, after MitraClip leaflet grasping for dynamic secondary MR, it is important to drive the blood pressure up to assess the severity of residual MR. As often during MitraClip procedures, patients are anaesthetised and well diuresed, resulting in a degree of MR that is not reflective of the awake and ambulatory states. If necessary, clip adjustments or additional clips may be required to address the geometric alterations from the increase in afterload.

In the appropriate clinical context, dynamic MR is an important consideration in patients with recurrent acute pulmonary oedema. Our case demonstrated the successful use of percutaneous edge-to-edge repair of the mitral valve with the MitraClip system to effectively address symptomatic dynamic MR in a critically ill patient at prohibitive surgical risk.

\section{REFERENCES}

1. Lancellotti P, Gérard PL, Piérard LA. Long-term outcome of patients with heart failure and dynamic functional mitral regurgitation. Eur Heart J 2005;26:1528-32.

2. Szymanski C, Levine RA, Tribouilloy C, et al. Impact of mitral regurgitation on exercise capacity and clinical outcomes in patients with ischemic left ventricular dysfunction. Am J Cardiol 2011;108: 1714-20.

3. Lapu-Bula R, Robert A, Van Craeynest D, et al. Contribution of exercise-induced mitral regurgitation to exercise stroke volume and exercise capacity in patients with left ventricular systolic dysfunction. Circulation 2002;106:1342-8.

4. Bertrand PB, Schwammenthal E, Levine RA, et al. Exercise dynamics in secondary mitral regurgitation: pathophysiology and therapeutic implications. Circulation 2017;135:297-314.

5. Masumoto A, Kubo S, Ohya M, et al. MitraClip therapy for dynamic mitral regurgitation with repetitive heart failure exacerbation. JACC Cardiovasc Interv 2019; $12: \mathrm{e} 215-7$.

6. Duino V, Fiocca L, Musumeci G, et al. An intriguing case report of functional mitral regurgitation treated with MitraClip. Medicine (Baltimore) 2015;94:e608.

Ningyan Wong, ${ }^{1} M B B S$, Wei Chieh Jack Tan, ${ }^{1} M B B S$, Wishnu Aditya Widodo, ${ }^{2} M D$, Biauw Chi Ong, ${ }^{3,4}{ }_{M B B S}$, Zee Pin Ding, ${ }^{1}$ MBBS, See Hooi Ewe, ${ }^{1}$ MBBS (Hons), Hak Chiaw Tang, ${ }^{1}$ MBBS, Khung Keong Yeo, ${ }^{1}$ MBBS

\footnotetext{
Department of Cardiology, National Heart Centre Singapore, Singapore

${ }^{2}$ Department of Cardiology, Jakarta Heart Center, Indonesia

${ }^{3}$ Department of Anaesthesiology, Singapore General Hospital, Singapore

${ }^{4}$ Department of Anaesthesiology, Sengkang General Hospital, Singapore

Correspondence: Dr Ningyan Wong, National Heart Centre Singapore, 5 Hospital Drive, Singapore 169609.

Email: wong.ningyan@singhealth.com.sg
} 